\title{
Interférences
}

Ars scribendi

$7 \mid 2014$

Le savoir sur la langue

\section{La signification d'une science platonicienne du langage selon Jean-Toussaint Desanti dans son cours inédit « Être et relation chez Platon »}

\section{Alain Gigandet}

\section{(2) OpenEdition}

Journals

Édition électronique

URL : http://journals.openedition.org/interferences/4645

DOI : 10.4000/interferences.4645

ISSN : 1777-5485

Éditeur

HiSoMA - Histoire et sources des Mondes antiques

\section{Référence électronique}

Alain Gigandet, « La signification d'une science platonicienne du langage selon Jean-Toussaint Desanti dans son cours inédit «Être et relation chez Platon » », Interférences [En ligne], 7 | 2014, mis en ligne le 11 décembre 2014, consulté le 15 septembre 2020. URL : http://journals.openedition.org/ interferences/4645; DOI : https://doi.org/10.4000/interferences.4645

Ce document a été généré automatiquement le 15 septembre 2020.

Tous droits réservés 


\title{
La signification d'une science platonicienne du langage selon Jean-Toussaint Desanti dans son cours inédit « Être et relation chez Platon »
}

\author{
Alain Gigandet
}

1 Jean-Toussaint Desanti, né en 1914 et disparu en 2002, a marqué la philosophie des sciences et l'épistémologie, et plus généralement l'enseignement et le débat philosophiques en France dans la seconde moitié du xxe siècle. Résistant, membre du Parti communiste français qu'il quitte en 1956, il est revenu bien plus tard, dans une perspective philosophique, sur son expérience politique (Un destin philosophique, 1982). Desanti a publié, entre autres: Les idéalités mathématiques (1968); La philosophie silencieuse ou critique des philosophies de la science (1975), Le philosophe et les pouvoirs (1976), Réflexions sur le temps (1992). Il se réclame de la tradition phénoménologique, dont il met en œuvre la méthode de façon extrêmement personnelle.

2 Helléniste et latiniste accompli, Desanti était un fin connaisseur de la philosophie antique, des présocratiques à saint Augustin, sur laquelle il est revenu tout au long de sa vie tant dans ses cours que dans ses écrits. Son cours de DEA et doctorat «Être et relation chez Platon", donné d'octobre 1986 à avril 1987 à l'Université Paris 1 Panthéon-Sorbonne en tant que Professeur émérite, non seulement est resté inédit, mais, à ma connaissance, n’a pas été réutilisé par son auteur, même partiellement, dans des publications ultérieures.

3 Le but de cette communication est de présenter la section du cours qui concerne le Cratyle, et plus précisément sa première partie, dans laquelle Desanti s'emploie à préciser l'objet de ce dialogue et sa place, décisive, dans l'économie de l'enquête platonicienne sur l'« être véritable». Pour ce faire, je me bornerai à " encadrer » de 
larges citations du cours, laissant ainsi entendre, autant qu'il est possible, la voix même de Desanti.

\section{Projet du cours}

4 L'idée de Desanti, que résume le titre du cours, est que la mise en crise de la théorie des Formes dont témoigne le Parménide a conduit Platon à repenser les fondements de sa doctrine de l'intelligible, en substituant à une ontologie des objets ou des réalités une ontologie de la relation. Ce programme, accompli dans le Sophiste, le Philèbe, le Politique, trouve un point d'appui et comme un modèle dans la théorie du langage, plus précisément dans l'analyse du nom dans sa fonction signifiante, élaborée au préalable dans le Cratyle. En d'autres termes, le Sophiste va exploiter, sur le plan ontologique, une structure construite à propos du langage par le Cratyle. Desanti parle, à propos de la relation entre les deux dialogues, d'une "homologie problématique ", autour de l'idée de sumplokê, d'entrelacement ${ }^{1}$.

\section{Détour par la Lettre VII}

5 Pour mener à bien ce projet, la première étape qui s'impose consiste en une élucidation de la théorie linguistique du Cratyle. Mais le Cratyle est un dialogue abrupt, au contexte en partie masqué. Pour reconstituer son horizon, celui de la préoccupation philosophique qui pousse Platon dans la direction d'une théorie du langage, Desanti propose de passer préalablement par la Lettre VII et sa célèbre "digression philosophique ».

6 La «digression» en effet désigne le nom, onoma, et la définition ou «formule expressive ", logos, comme les deux premiers des cinq éléments permettant d'acquérir la science de tout être, quel qu'il soit. Mais la «digression» est-elle vraiment digressive? Desanti insiste sur le fait qu'elle est rigoureusement articulée au récit de l'échec politique et philosophique de Platon à Syracuse, qu'elle en constitue en fait le point de convergence. Donnons-lui la parole ${ }^{2}$ :

Or, dans cette Lettre VII, il s'agit bien de définir des modes d'action adéquats concernant la cité. Le sens de cette « digression » y est suspendu. Ce projet suppose toujours, quant à sa réalisation, des procédures de persuasion: parler, dire, légiférer (graphein, écrire, inscrire). Il faut avoir affaire aux noms, aux logoi, aux images - que cela soit visible. Si la philosophie est le point d'aboutissement de l'éducation du citoyen en vue d'établir de bonnes lois, si elle concerne la formation de la psukhê, l'opération par quoi les âmes des citoyens sont "criblées" par la bonne loi, alors il faut mettre en jeu dans la philosophie les noms, les phrases, etc. Dans cette opération, ce que l'âme apprend (selon la pureté de sa race ontologique), c'est à parcourir tout ce champ constitué par noms, images, etc., de manière à reconnaître sa parenté avec le cinquième élément. Se servir de ce qui est le plus éloigné du cinquième élément pour s'en découvrir proche. C'est cette exigence qui fait qu'il est illusoire de vouloir écrire la philosophie, parce que là, on est astreint, asservi aux mots, phrases, images. Que faire? Il faut circuler, "frotter les uns contre les autres » ces éléments, sans repos, et par là découvrir pour soi-même (et communiquer aux autres) la relation substantielle et originaire de l'âme à l'être. Le polylogue, la diatribê, l'échange des âmes parlantes. Voir 343d-e : si dans l'ordre de la descendance on est situé au bon rang, si l'on n'a pas l'âme trop lourde, on peut, en frottant sans se lasser les éléments les uns contre les autres, approcher la 
constitution de la science.

Quelle est cette science? Epistêmê est un terme très présent chez les contemporains de Platon (epistêmê kai technê). C'est la géométrie dans le style d'Eudoxe; c'est l'astronomie des sphères homocentrées, d'Eudoxe aussi; c'est la médecine hippocratique; également la musique et la danse, qui reposent sur le mouvement en harmonie.

Elle s'inscrit dans le monde des mots, des phrases, des images, dévoyée en polumathia par les sophistes comme Hippias. En fait, on peut isoler deux versions antagonistes de la polymathie :

- a. celle des sophistes, qui transmettent les savoirs tels qu'ils sont constitués, investis dans le champ du savoir productif de pouvoir, de profit, etc. ;

- b. celle de Platon, qui rend capable de produire des législateurs et non des avocats d'opinion.

La science comme quatrième élément, c'est cette epistêmê réformée par la sungeneia, élément de l'epistêmê. Celle-ci a donc le noûs comme parent, et c'est cette parenté qui invalide l'écrit, et non le connaître par la méthode du polylogue, à condition toutefois que l'objet soit le bon, et l'âme la bonne.

Ce qui renvoie à la situation du langage chez Platon. Il y a un arbitraire dans les noms, mais qui ne change rien à leur pouvoir de désignation. Il faut se rapporter au nom, sans s'y fier. Dans le Cratyle, les thèses d'Hermogène et de Cratyle sont renvoyées dos à dos, mais en maintenant l'exigence que, quelle que soit la nature des noms, ceux-ci visent la désignation véritable. Le nom comme profération est de l'entre-deux, être non-être, mais il est nécessaire. Il faudra donc poser le problème de la rectitude des noms, c'est un moment de ce frottement par quoi l'on approche du cinquième élément.

À partir de là, plusieurs chemins se présentent. Il y a des dialogues qui essaient, dans tous les sens, les hypothèses concernant une proposition. C'est le Parménide, à rapprocher à cet égard du Tractatus de Wittgenstein. Avec un non-résultat apparent, quoique les signes de la solution soient présents. Il y a le fil de la sungeneia : le Phédon, le Phèdre. Il y a le fil de l'epistêmê : la République, les Lois.

Desanti revient ensuite sur le mode ambigu de l'implication du langage dans la démarche de l'enquête dialectique, et ses raisons :

Cela ne va pas tout seul, cet enfantement donne bien de la peine. Ce parcours doit être le fait de quelqu'un de bien constitué, ayant une bonne nature [eu phusikon]. Alors la vie selon l'exigence du connaître engendrera la science. Encore faut-il que les partenaires aient été produits ou se trouvent produits de telle façon que leur relation soit fructueuse. Qui sont les partenaires? Plus loin, Platon dit «les hommes ". Mais qu'est-ce qui peut être dit constitué de bonne nature ? C'est le bien, to kalon, nommé ailleurs. Il y a concordance entre l'homme qui mène cette vie et la chose même dont il ressent douloureusement la séparation, et le désir de s'y unir. Qu'est-ce qui, en revanche, est exclu de la «bonne nature »? Dans ce mouvement d'aller-retour, il peut y avoir confusion des signes, il peut se faire que ce qui est par soi-même le but du connaître soit masqué par ce qui n'est qu'un moment du cheminement. Alors le mouvement de la diagogê est dévié : il se poursuit, mais vers autre chose - non la connaissance, mais le triomphe sur l'adversaire. Cette déviation est même inévitable $d u$ fait qu'on vit en cité. Voir l'intermède $d u$ philosophe du Théétète qui veut «fuir dans la clarté », fuite qui se paie du mépris de la foule - jeu de la réussite et de l'échec... Seule l'insistance mise sur la direction, la constance vers la cible peut éviter cela, mais il y faut de la peine.

La menace vient donc des étapes mêmes qu'il faut parcourir. Il y a là une théorie des leurres: le bonheur, le plaisir, la tyrannie, l'écriture de livres à prétention philosophique. C'est pourquoi le thème majeur de la Lettre VII est intimement présent dans la prétendue digression : il s'agissait en effet pour Platon d'expliquer son échec politique. Dion s'est trompé de partenaires : ce n'étaient que des crapules qui désiraient le pouvoir et non la réforme des institutions. Errance, donc, et 
confusion des signes. Les mêmes qui interdisent l'engendrement de la science interdisent celui du juste et de la bonne constitution.

[...] Il reste que se manifeste toujours la différence entre les mortels et l'être, comme tension, désir, Erôs. Mais parenté [sungeneia], ressemblance [homotês] sont aussi dans cette distance. Le plus lointain est le plus proche. La proximité se manifeste dans le dialogue, dans l'insatisfaction que j'éprouve à ce qui se présente l'insatisfaction portée par l'être. Dia-gôgê, c'est un écart que l'on doit traverser, et qui ne se comble pas de lui-même.

On privilégiera donc : onoma, epistêmê, logos, alêtheia.

Ce que les choses sont, c'est à dire. C'est objet de langage, et la dialectique doit apprendre à le dire. Par conséquent, on a affaire à des noms, des verbes, afin d'articuler dans une langue à la fois désignative et communicative. Le logos se produit dans l'usage du langage, il faut donc se mettre au clair avec la question du langage: c'est l'objet du Cratyle. Dans ce dialogue, ce qui est en jeu, c'est la possibilité d'utiliser à bon droit le langage ; c'est une fondation de l'exigence de sa médiation, la médiation du parler.

Ensuite, il faudra fonder la logique catégoriale de l'être : c'est l'objet du Sophiste.

Entre ces deux dialogues s'établit une homologie problématique. Dans les deux cas, l'interrogation porte sur l'entrelacement [sumplokê]. Dans le Cratyle, c'est l'entrelacement des mots, des phrases (question du sens). Dans le Sophiste, c'est l'entrelacement des formes (le mouvement et le repos, l'être et le non-être). Ce que Platon met en place, c'est une théorie de la relation, qui utilise le paradigme du tissage (la trame, la chaîne) et qui concerne le mode de vie, la vie propre du parler et du signifier (Cratyle), la vie de l'intelligible lui-même (Sophiste).

Problème que pose le Cratyle : pourquoi le langage demande-t-il à être examiné ? Et pourquoi cette question sur la naturalité ou la conventionalité du mot? En fait, Platon propose une solution négative: le langage n'est ni l'un ni l'autre. Il est iconique au sens de Peirce, d'une autre nature que l'être, mais qui lui ressemble. C'est ce qui autorise l'usage du langage pour viser l'être.

Il faut examiner l'exercice de la parole chez les contemporains de Platon. Du langage poétique, on est passé à celui de l'orateur et du logographe.

\section{Situation du Cratyle}

Desanti explique donc que, pour Platon, élucider la nature du nom et de l'entrelacement des noms dans l'énoncé constitue la question préalable de la philosophie. En dépit de ses bizarreries, c'est au Cratyle que revient le privilège de prendre cette question en charge. La manière même dont il le fait, à partir du dispositif à trois (Hermogène, Cratyle, Socrate) et en mobilisant le paradigme technique, c'està-dire en suspendant la question de la nature du nom à celle de son usage, tout cela engage non seulement les fondements de la «linguistique " platonicienne, mais ceux d'une épistémologie et d'une ontologie encore à venir (le cadre problématique dans lequel les enquêtes successives seront mises en œuvre).

\section{La relation à trois et les enjeux du débat sur le nom}

Laissons à nouveau parler Desanti :

Le Cratyle repose sur une relation à trois :

- a. Hermogène (les noms sont établis nomô) ;

- b. Cratyle (ils ont une rectitude phusei), chacun, donc, en possession d'une thèse ;

- c. Socrate, qui cherche.

Dans cet entre-deux, Socrate va chercher son chemin. Et, à la fin, il laissera la 
réponse indécise après avoir frotté les opinions les unes contre les autres. Mais un projet se dégagera de tout cela : quel que soit le parti que l'on prend, l'usage de la parole doit concerner l'ousia. Le dialogue est apparemment aporétique, mais obliquement, il est positif. Comme souvent chez Platon, c'est la voie oblique qui parvient à des réponses. Ces solutions obliques sont fondamentales.

- Exemple 1: le Parménide, qui comporte deux parties: (a) une critique féroce par Platon de sa propre théorie des Formes, qui conduit à une aporie; (b) un examen dialectique, presque éristique, en neuf hypothèses, de la thèse "le multiple est l'un ». Tout cela ne donne lieu à aucune conclusion apparente. Cependant, il y a des hypothèses qui, sans privilège apparent, ont celui de s'articuler avec d'autres thèses $\mathrm{du}$ platonisme. Par exemple l'hypothèse $\mathrm{n}^{\circ} 4$, qui envisage l'un dans le multiple : ce sera la voie du Sophiste, du Philèbe ou du Politique. Une solution oblique.

- Exemple 2 : le Théétète, aporétique aussi, mais la démarche qui permet de parvenir à la définition finalement écartée côtoie négativement le savoir, le fait apparaître en creux. La science n'est ni l'opinion, ni la sensation, elle suppose la fuite hors $\mathrm{du}$ devenir et ainsi de suite.

Qu'en est-il donc de cette "dramatisation"? Il faut revenir à la Lettre VII : le problème n'est pas d'enseigner la science mais de mettre chacun en mesure de la pratiquer. C'est le chemin difficile de destruction des opinions, dans lequel la pensée de chacun doit trouver son assise. L'embarras mène vers les chemins de traverse.

Pourquoi poser la question du rapport nomô/phusei à propos des onomai? Le choix des personnages est significatif.

D'abord Cratyle, héraclitéen. Platon a peut-être suivi ses leçons.

Hermogène, lui, est du côté des sophistes: le langage est pris comme objet, thématisé, il est analysé comme un outil, un objet en vue de...

Or la philosophie, au sens où nous l'entendons, naît dans cet entre-deux, dans ce champ borné par l'utilitarisme sophistique d'une part, par le poids ontologique des mots d'autre part. De l'être au langage, distance maximale; de l'être au langage, distance minimale. Pour la sophistique, la maîtrise des mots permet la maitrise des opinions, ce qui importe, c'est de conclure - vrai ou faux, cela en revanche n'importe pas. Technique d'organisation des croyances et de maîtrise des pratiques. Avec Héraclite, le terrain est ontologique, le mot est la chose même. «Ce n'est pas moi qui parle, c'est le logos ». C'est aussi le langage de Parménide. Voir le début du poème de Parménide : une invocation à la déesse, où l'initié a la révélation des deux voies dans le langage de la divinité. Dans la voie du vrai, la parole demande à être reçue comme vraie. Le langage révèle, qui comprend le mot comprend la chose et cela demande à être cru.

Cet affrontement du vrai et de l'artifice dégage un entre-deux où Platon va frayer sa voie. À cette époque, l'alêtheia propre aux mots s'éloigne, n'est plus reçue dans la cité : on ne croit plus à la révélation (voir Détienne, Les maîtres de vérité en Grèce archaïque). On se souvient de l'oracle de Delphes, qui ne dévoile ni ne cache, mais qui montre les signes (sêmainein) : c'est l'assise de ce discours. Or voilà que la parole violente, de maîtrise, autorisée par la voie même du vrai, provoque une désadhésion : de là la révolution sophistique. Et à l'intérieur de cette révolution, la philosophie reprend naissance. Le philosophe ne peut plus dire simplement « voilà ce qui est», il faut qu'il ajoute «et si c'était autre chose?» Nécessité des articulations. Le logos est devenu profane, spéculatif, avant d'être expressif de l'être par la vertu d'une position d'énonciation (le fait d'être proféré par certains en certaines circonstances). Il faut faire un deuil, et déterminer le bon usage du langage.

La symbolique des personnages du Cratyle, avec l'opposition nomô/phusei, va courir dans la réflexion grecque au moins jusqu'aux stoïciens. Ici, l'opposition est une façon de prendre conscience de la situation historique du langage à l'époque de Platon, et de tracer une problématique du rapport des mots aux choses. Comment faut-il parler pour dire vrai de l'être? Réponse: à partir du grec - d'où les 
étymologies (voir le Ménon et la question de Socrate concernant le petit esclave). Mais il y a de la dérision là-dedans. Il ne suffit pas de parler grec.

manière suivante :

Contre Hermogène, on feint d'établir un rapport naturel mimétique nom/chose et on se laisse guider par l'hypothèse que le nom a affaire à l'essence de la chose. Tout le passage est quelque peu une parodie de la position cratylienne.

Ce rapport en définitive est dans une distance fondamentale à l'essence. Il va falloir faire cet aveu, ou plutôt l'arracher au langage lui-même.

Cratyle est héraclitéen, Platon doit faire son chemin derrière Héraclite. Derrière Cratyle, on lit Protagoras, l'homme-mesure, l'adéquation du langage au flux des choses. Les noms sont-ils phusei ou nomô ? À l'horizon de cette question, il y a celle de la phusis : qu'est-ce qu'elle est?

Hermogène appartient au groupe des sophistes de l'espèce enseignante, dont la figure dominante est Prodicos (noter l'allusion de Socrate aux séances payantes).

Socrate va pousser à l'extrême, exténuer leur désaccord. Derrière cela, son projet politique de domestiquer le langage en vue de la politeia, de la sophia, de l'epistêmê. Il a son idée sur la nature du langage : elle doit avoir rapport avec l'ousia.

Ce que cherche Socrate, c'est le chemin de l'usage réglé du langage, dans cet espace de discussion ouvert par la sophistique. Construire l'orientation de la mise en pratique de l'usage du langage en vue du dévoilement-développement du vrai.

Conclusion suggérée par le Cratyle : cet usage suppose à la fois mimêsis et distance. Mais il y a une mauvaise mimêsis, celle de la relation modèle/portrait, ainsi qu'une mauvaise distance, celle qui laisse le mot libre de son rapport à la chose. C'est pourquoi il faut mettre en œuvre le législateur (nomothetês, avec jeu de mot sur nomos), qui va construire l'usage droit en tant que dialecticien.

Hermogène et Cratyle, en définitive, seront renvoyés dos-à-dos, mais on aura fait l'épreuve. Il y a un déséquilibre dans le dialogue: Socrate s'acharne à réfuter la conventionalité, mais de telle façon que l'étayage démonstratif de la thèse cratylienne frise la réfutation. Il y a parodie du cratylisme.

\section{Linguistique platonicienne}

11 Soit maintenant à faire ressortir les linéaments d'une "linguistique platonicienne ». Platon veut montrer à quel point la langue grecque a pénétré le réel, naturel et culturel : tout ce qui est trouve son point de réflexion dans le langage. Renvoi universel, grand miroir. Plutôt, Platon feint d'interpréter ce renvoi comme miroir, mais c'est humoristique. Ce qui est en jeu dans le grand catalogue étymologique, c'est l'enracinement du monde grec dans la langue grecque. Elle dit tout cela. Le langage est legein, il a pouvoir de véhiculer cet ensemble de significations, et c'est ce pouvoir qui est affirmé dans ce déploiement d'étymologies fantaisistes.

Dès le prologue, l'affrontement des deux personnages ouvre le champ de la recherche, il le norme. La sophistique, l'héraclitéisme, et l'ombre de la sophia, Socrate, qui prend sur lui l'insuffisance des deux débatteurs. La voie, c'est l'affirmation de la norme, la recherche du bon usage, qui cependant n'effacera jamais la distance.

Critique de la thèse d'Hermogène : deux moments sont à distinguer.

Le premier moment donne lieu à trois démarches pour établir le rapport du nom à l' ousia :

- a. 385a-e : les dénominations peuvent être correctes ou incorrectes (qui confond les noms confond les choses) ;

- b. 385e-386a : les choses, ta pragmata, renvoient elles-mêmes à une essence stable ; 
- c. 386b-391a : les actes qui concernent les choses sont comme les choses mêmes. On doit agir conformément à ce que la chose montre, exige.

Conséquence: qu'est-ce que nommer? une opération effectuée dans l'usage de la parole, pour l'usage de la parole. Parler, c'est accomplir un acte, qui, comme tout acte, doit avoir ses conditions de stabilité. Alors il y a une rectitude des noms, et quelqu'un se sert fondamentalement des noms, c'est le dialecticien.

Dans le second moment, on se demande en quoi consiste cette rectitude. Examen de la structure des noms, dans son rapport de convenance, mimétique, à leur référent. Mais on va montrer ensuite que, s'il y a un bon usage du nom, il ne peut s'appuyer sur ce rapport mimétique. Ce point fera l'objet d'une élucidation complète dans le Sophiste: le nom identique à la chose rend impossible de parler de quoi que ce soit.

Desanti s'attache ensuite plus précisément à l'étude du texte qui va de $387 \mathrm{c}$ à $390 \mathrm{~d}$ :

Il s'agit du dernier moment d'un mouvement dialectique qui met en cause la thèse d'Hermogène. Il se conclut par une hypothèse dont on tentera ensuite de vérifier la validité dans un champ d'exemples, les «fausses étymologies ». La direction en est la suivante :

- a. établir les noms est une activité réglée, de l'ordre de l'ergon; ce n'est pas un «travail», mais une activité qui a affaire aux choses (voir l'opposition connue ergodê/logodê, « en actes/en paroles », c'est-à-dire « en réalité/en apparence »);

- b. étant ergon, l'établissement des noms est sous la responsabilité d'un "acteur ", un responsable qui sera nommé à la fin "législateur». Ce dernier opère sous un double contrôle, d'abord des choses elles-mêmes, puis de celui qui a éminemment affaire au langage, le dialecticien. Le législateur est-il le dialecticien lui-même ? Cela est laissé dans l'ombre. Qui est législateur? Peut-être l'homme lui-même, en revenant à la Lettre VII.

Remontons aux deux mouvements précédents.

- en 385 : on ramène la thèse d'Hermogène à celle de Protagoras (anticipation de la critique développée dans le Théétète) ;

- en 386 : si Protagoras a tort, les choses ont une essence stable, et il doit en être ainsi également des actions qui concernent les choses.

En 387b, question de Socrate : «Et parler (to legein), n'est-ce pas aussi un acte (mia tis tôn praxeôn estin) ? - Oui ». C'est un axiome, cela ne sera pas discuté. Parler est une action. Or cela demande réflexion. Il faut revenir au tout début du dialogue, qui est très abrupt, sans prologue comme en comportent la plupart des autres dialogues. C'est qu'il y a un dialogue derrière l'arrivée de Socrate. On se disputait, et on commence à s'inquiéter: Hermogène et Cratyle sont des gens consubstantiellement en dialogue, ils sont en proie au discours et ont l'expérience du parler.

Par ailleurs, la question est surdéterminée à cause du mot praxis. On est dans le domaine de l'action orientée, en tant qu'elle aboutit et qu'elle est susceptible d'atteindre son but. Ce qui la définit comme praxis, c'est la bouleusis, le vouloiratteindre. À travers elle, la praxis vise ta pragmata, les affaires communes, autrement dit la délibération, l'étude, la gymnastique, la guerre. Le legein est donc une des praxeis, ce qui signifie que, sans la parole, nous, hommes de la cité, nous ne pouvons pas envisager un but possible. Mais de la parole envisagée intrinsèquement, y a-t-il but, et lequel ? Ce n'est pas clair.

Quoi qu'il en soit, cet accord sur l'axiome premier entraîne tout le reste. Socrate : « Est-ce donc d'après une opinion quelconque sur la façon dont on doit parler qu'on parlera correctement? » An tô dokê : d'après n'importe qui pouvant parler, tel qu'il est pris dans le champ de la doxa, « au gré de ses croyances changeantes ». À quelle condition parlera-t-on d'une façon droite, orthôs? L'usage incorrect, cela peut être précisément celui qui détourne le langage de ses fonctions, ou détourne l'usage pour transmettre ses croyances non vérifiées, ou encore pervertit la fonction de désignation univoque des phrases, en assemblant les mots n'importe comment. 
Obliquement, le texte introduit la dichotomie entre le champ de la doxa qui, si on s'y tient, autorise toutes les perversions du langage, y compris dans la désignation; et le champ des pragmata, qui ont une réalité stable et ne supportent pas ces jeux. On a affaire sérieusement à ce que concerne le langage comme activité : le monde, les dieux, la cité. On ne peut pas faire n'importe quoi avec le legein. Faute de quoi on se joue de tout cela et on transgresse la règle du langage.

Legein, c'est donc s'inscrire dans ta pragmata.

C'est seulement si l'on ne se rapporte pas au champ de l'opinion, si on ne se laisse pas porter par ses croyances, que l'on obtiendra satisfaction quant à ce qui est le souci de l'homme qui agit (poien). En se pliant, dans sa parole, à la chose qu'il laisse venir à la façon dont elle est, celui qui parle obtiendra satisfaction (poiêsei : ça marchera pour lui) et il s'exprimera, c'est-à-dire que sa parole sera pleine et reçue, on l'entendra. S'il ne fait pas cela, il commettra une faute et il n'obtiendra rien.

De quoi s'agit-il, en somme? "Toi, Hermogène, tu écris des discours pour les sophistes. Comment procèdes-tu? Tu dois employer les mots comme il faut. » Il y a là plusieurs niveaux de sens :

- a. faire reconnaître à Hermogène l'implicite présence du langage ;

- b. lui suggérer que si le langage glisse tout entier dans le champ de la doxa, il en va des choses elles-mêmes, on ne saura plus ce qui est utile ou nuisible, la cité sombrera.

Ce premier moment de la section du cours consacrée au Cratyle s'arrête donc au seuil de la partie technique de la discussion, qui s'organise autour de la question : «qu'est-ce que nommer ${ }^{3}$ ? " Je rassemblerai pour conclure les trois axes de lecture fondamentaux qui me paraissent ordonner l'analyse que Desanti consacre à la place du Cratyle dans l'économie de l'enquête platonicienne sur l'être véritable. En premier lieu, montrer que l'élucidation du langage, centrée sur l'activité de dénomination, constitue une priorité pour l'entreprise dialectique platonicienne, sommée ainsi de ressaisir ce qui constitue pour elle une condition fondamentale. Ensuite, éclairer le contexte culturel et intellectuel qui justifie le choix des interlocuteurs, Cratyle et Hermogène: "désacralisation du langage", essor de la sophistique. Enfin, restituer les enjeux politiques fondamentaux qui donnent à cette enquête son horizon premier : la cité en tant qu'elle a partie liée avec un certain «régime de langage» et la nécessité de comprendre la pathologie qui l'affecte.

\section{BIBLIOGRAPHIE}

DESANTI J.-T. 1968, Les idéalités mathématiques. Recherches épistémologiques sur le développement de la théorie des fonctions de variables réelles, L'ordre philosophique, Paris.

- 1975, La philosophie silencieuse ou critique des philosophies de la science, L'ordre philosophique, Paris.

- 1976, Le philosophe et les pouvoirs. Entretiens avec Pascal Lainé et Blandine Barret-Kriegel, Ordre des choses, Paris.

- 1982, Un destin philosophique, Figures, Paris.

- 1992, Réflexions sur le temps. Conversations avec Dominique-Antoine Grisoni, Figures, Paris. 
DÉTIENNE M. 1967, Les maîtres de vérité dans la Grèce archaïque, Textes à l'appui, Paris.

SOULEZ A. 1991, La grammaire philosophique chez Platon, Philosophie d'aujourd'hui, Paris.

\section{NOTES}

1. Voir depuis, entre autres, Soulez 1991.

2. Les citations qui forment le corps de cet article sont issues de mes propres notes de cours, avec la marge d'imprécision que cela comporte inévitablement par rapport à la parole originale.

3. La suite logique de ce texte doit consister en une étude du commentaire que Desanti, dans un second temps, consacre à l'analyse platonicienne de l'acte de dénomination et de la nature du nom.

\section{RÉSUMÉS}

Dans son cours intitulé « Être et relation chez Platon» (1986-1987), Jean-Toussaint Desanti montre les raisons qui font que l'enquête dialectique sur la vérité de l'être chez Platon demande à ce qu'on élucide en préliminaire la question de la nature du langage et explique comment le Cratyle est pensé pour fournir cette élucidation. La construction du dialogue par Platon est éclairée par des références à l'arrière-plan historique, politique et intellectuel du débat sur la nature et l'usage du langage.

In his course of 1986-87 entitled "Être et relation chez Platon", Jean-Toussaint Desanti shows the reasons why Plato's dialectic enquiry upon "true beeing" needs a preliminary elucidation of the nature of language and explains how the Cratylus is meant to provide it. Plato's making of this dialogue is clarified by referring to the historical, political and intellectual background of the debate upon the nature and the use of language.

\section{INDEX}

Keywords : theory of language, words and things nomsmotscles Eudoxe de Cnide, Héraclite d'Éphèse, Hippias, Hippocrate, Platon, Prodicos Mots-clés : théorie du langage, mots et choses Index géographique: Delphes, Syracuse

\section{AUTEURS}

\section{ALAIN GIGANDET}

Université Paris-Est Créteil 